\title{
Heat Transfer in Hydromagnetic Flow over an Unsteady Stretching Permeable Sheet
}

\author{
Susheela Chaudhary \\ Department of Mathematics \\ Government Science College, Sikar, 332001, Rajasthan, India \\ E-mail: susheelamaths@gmail.com \\ Santosh Chaudhary \\ Department of Mathematics \\ Malaviya National Institute of Technology, Jaipur, 302017, Rajasthan, India \\ Corresponding author: d11.santosh@yahoo.com \\ Sawai Singh \\ Department of Mathematics \\ Government Science College, Sikar, 332001, Rajasthan, India \\ E-mail: dhayal.sawaisingh@yahoo.com
}

(Received January 28, 2019; Accepted May 16, 2019)

\begin{abstract}
Unsteady laminar boundary layer flow of viscous incompressible electrically conducting fluid along a continuous stretched permeable surface with the magnetic field effect is investigated. The defining characteristics of unsteady laminar boundary layer flow are governed a more than one independent variables, stretching velocity and surface temperature of the field. Governing equations are obtained for influencing parameters and transformed into ordinary differential equations by taking convenient similarity variables. Runge-Kutta fourth order method in corporation by the shooting technique is introduced to carry out numerical computations of the investigation. Velocity and temperature profiles are computed and represented graphically for the influences of suction/injection parameter, unsteadiness parameter, magnetic parameter and Prandtl number, while numerical solutions of local skin friction coefficient and local Nusselt number are discussed through tables. For non-magnetic condition, results are found in concordance with earlier research work.
\end{abstract}

Keywords- Heat transfer, Hydromagnetic, Unsteady flow, Stretching permeable sheet.

\section{Introduction}

Studies of heat transfer and hydrodynamic flow towards a stretchable sheet have been receiving substantial importance over to its roles in upgrading technological applications and growing industrial processes. These types of problems have numerous characterizations with the small velocities in areas of geothermal where the layers of the shallow sheet are being stretched. The concept of stretching has useful applications in the hydrothermal system where fluid properties at depth influence the geothermal energy work. This study has value in important engineering applications such as drawing, tinning, and annealing of copper wire, crystal growing, etc. in the area of metallurgy and chemical engineering procedures. The above mentioned applications affect the continuous filaments cooling or strips via drawing them by a quiescent liquid. Besides, it has widespread applications in geophysics and thermal science, particularly in the packed bed reactors, petroleum resources recovery, geothermal energy technology, building thermal insulation, and underground disposal of chemical and nuclear waste. The final product quality is influenced by the 
International Journal of Mathematical, Engineering and Management Sciences

Vol. 4, No. 4, 1018-1030, 2019

https://dx.doi.org/10.33889/IJMEMS.2019.4.4-081

heat transfer rate at the stretching wall. Sakiadis (1961) initiated the boundary layer flow over a moving continuous solid surface with constant velocity. Further, Crane (1970) extended it and obtained an effective analytical solution for the steady two-dimensional boundary layer flow problem in a quiescent incompressible fluid caused through the elastic flat surface stretching which acts with it plane over linearly varying surface speed. Many researchers such as Andersson (2002), Hayat and Sajid (2007), and Mukhopadhyay and Layek (2012) have instigated the problems of steady flow due to a stretching sheet in different conditions. Recently, Chaudhary and Choudhary (2018), and Ram et al. (2018) have conducted numerous analysis on the stretching surface problems in steady boundary layer flow.

The findings of the above studies that deal with stretching sheet are more significant with considering that the flow conditions are to be steady. In the unsteady flow condition where flow vary with respect to both time and space, the stretchable plate can vary along time and thus the appearance of unsteady stretching surface grow into practical problems. To enhance the physically realistic applications of the flow situation, that is worthwhile to carry unsteadiness in the governing equations of fluid flow analysis. A considerable number of engineering problems such as startup process and periodic fluid motion are defined by unsteady boundary layer conditions. Wang (1990) was the first one who studies the hydrodynamics of an unsteady boundary layer fluid flow of a liquid film past a stretched surface. Many researchers such as Elbashbeshy and Bazid (2004), Ishak et al. (2008), Elbashbeshy and Aldawody (2010), Chaudhary et al. (2015), and Ram et al. (2017) are determined the problems of unsteady flow over a stretching plate in different situations.

Furthermore, the studies of heat transfer and flow along a permeable area are important in predicting the behavior of practical applications particularly in geophysical fluid dynamics like as small blood vessels, the human lung, wood, sandstone, limestone and beach sand. In polymer processing industries, the flow along a permeable stretching surface has extensive characterizations. The advancement in engineering and industrial applications of heat transfer and flow due to the permeable surface have a lot of attention by researchers. Beavers and Joseph (1967) were pioneered the exploration of the boundary condition at a naturally permeable wall. Many researchers such as Magyari and Keller (2000), Liao (2007), and Khader and Megahed (2014) are considered the permeable stretching surface flow problem under different situations. Recently, Chaudhary and Kumar (2015), Maity et al. (2016), and Ghadikolaei et al. (2018) are considered the heat transfer and flow over a stretched sheet in a porous medium and found the numerical solution.

Unsteadiness in hydromagnetic flow has received attention to some considerable extent in recent years due to the facts that numerical modeling of problems has developed a better understanding of practical and physical processes involved in engineering and technological applications. The field of solar energy collection, the design of cooling systems for electronic devices, an infinite metallic plate cooling in a cooling bath, a polymer plate or filament extruded continuously through a dye, geothermal reservoirs, heat exchangers, MHD marine propulsion, the boundary layer over a liquid film in condensation procedures, casting of magnetic-levitation and molten metal MHD stirring etc. are continuously driven by the influences of hydromagnetic unsteadiness of flow in variable situations. The problem of variable surface heat and mass flux of an unsteady natural convection MHD fluid flow over an inclined plate was presented by Jat and Chaudhary (2009), and Rashad (2014). Recently, hydromagnetic flow through unsteady stretching sheet was investigated by Mabood and Khan (2016), and Tian et al. (2017). Until, very recently, some scientists and 
International Journal of Mathematical, Engineering and Management Sciences

Vol. 4, No. 4, 1018-1030, 2019

https://dx.doi.org/10.33889/IJMEMS.2019.4.4-081

researchers like as Chaudhary et al. (2018), Dey (2019), Pandya et al. (2019), and Dutta et al. (2019) explored the fluid flow analysis with the magnetic impact.

With reference to above discussed applications and explorations, the primary objective of the present investigation is to expand the analysis of Ishak et al. (2009) for an electrically conducting fluid with the impact of the magnetic field associated to specified boundary conditions.

\section{Mathematical Model}

Two-dimensional unsteady laminar boundary layer flow of a quiescent viscous incompressible and electrically conducting fluid along a continuous stretched permeable sheet is assumed. The $x-$ axis is considered forward the continuous stretchable plate in the flow direction with the leading edge of the sheet as the origin, and $y$-axis is normal to it. The flow is assumed to be circumscribed in the upper half plane $y>0$. In the perpendicular direction of the stretching sheet, a uniform magnetic field of strength $H_{0}$ is applied as depicted in Figure 1. The induced magnetic field is negligible along to the magnetic Reynolds number is taken so small. The plate is impulsively stretched at time $t=0$, along $x$-axis with the stretching velocity $u_{w}(x, t)=\frac{a x}{1-c t}$, keeping the origin fixed in the fluid and surface temperature $T_{w}(x, t)=T_{\infty}+\frac{b x}{1-c t}$ where $a$ is positive constant, $x$ is the coordinate measured along the stretching sheet, $c$ is constant with $c \geq 0$ and $c t<1, T_{\infty}$ is the ambient temperature, $b$ is constants with $b \geq 0$, and both $a$ and $c$ have dimension time ${ }^{-1}$. All properties of the fluid are summarized to be constant all over the flow. Under these considerations along with the boundary layer approximations and neglecting the viscous dissipation, the basic equations that describe the case are as follows

$$
\begin{aligned}
& \frac{\partial u}{\partial x}+\frac{\partial v}{\partial y}=0 \\
& \frac{\partial u}{\partial t}+u \frac{\partial u}{\partial x}+v \frac{\partial u}{\partial y}=v \frac{\partial^{2} u}{\partial y^{2}}-\frac{\sigma_{e} \mu_{e}^{2} H_{0}^{2} u}{\rho} \\
& \frac{\partial T}{\partial t}+u \frac{\partial T}{\partial x}+v \frac{\partial T}{\partial y}=\alpha \frac{\partial^{2} T}{\partial y^{2}}
\end{aligned}
$$

with the boundary conditions

$$
\begin{array}{lc}
u=u_{w}(x, t), v=v_{w}(x, t), T=T_{w}(x, t) & \text { at } y=0 \\
u \rightarrow 0, T \rightarrow T_{\infty} & \text { as } y \rightarrow \infty
\end{array}
$$

where $u$ and $v$ are the velocity components in the $x$ and $y$ directions respectively, $v=\frac{\mu}{\rho}$ is the kinematic viscosity, $\mu$ is the coefficient of fluid viscosity, $\rho$ is the fluid density, $\sigma_{e}$ is the electrical conductivity, $\mu_{e}$ is the magnetic permeability, $T$ is the temperature of the fluid, 
International Journal of Mathematical, Engineering and Management Sciences

Vol. 4, No. 4, 1018-1030, 2019

https://dx.doi.org/10.33889/IJMEMS.2019.4.4-081

$\alpha=\frac{\kappa}{\rho C_{p}}$ is the thermal diffusivity, $\kappa$ is the thermal conductivity, $C_{p}$ is the specific heat at constant pressure, $v_{w}(x, t)=-\left(\frac{v u_{w}}{x}\right)^{1 / 2} S$ is the mass transfer at the surface with $v_{w}>0$ for injection and $v_{w}<0$ for suction, and $S$ is the suction parameter when $S>0$ or injection parameter when $S<0$. It allows to notice that at the time $t=0$ (initial motion), equations (1) to (3) defines the unsteady flow past a stretching sheet.

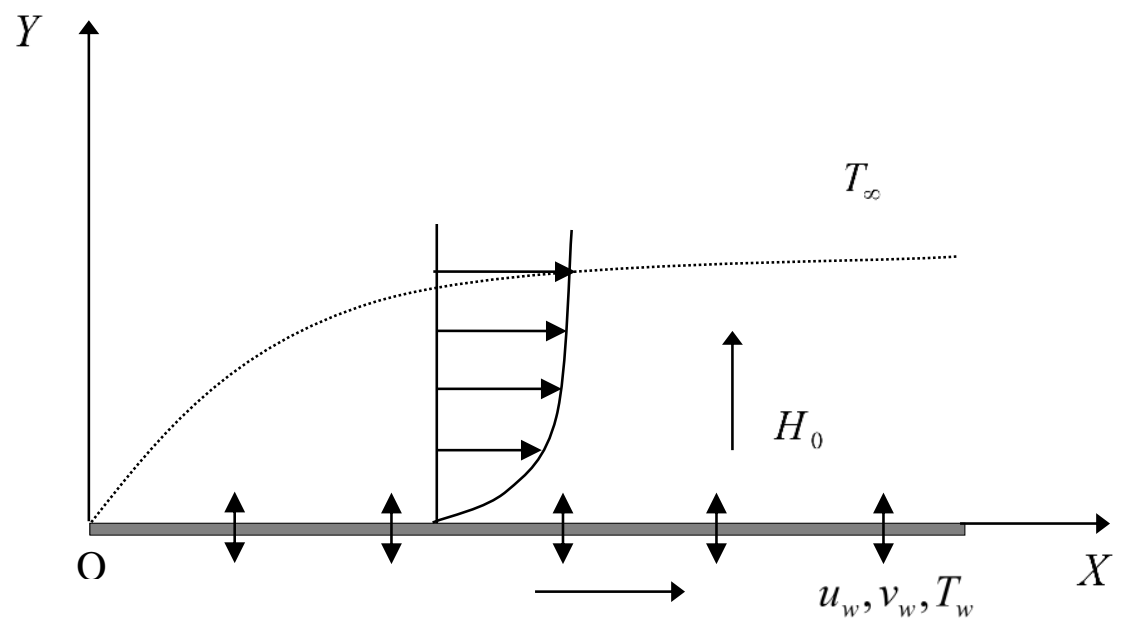

Figure 1. Flow geometry and coordinate system

\section{Similarity Variables}

The continuity equation (1) is identically satisfied through proposing a stream function $\psi(x, y, t)$ defined like that $u=\frac{\partial \psi}{\partial y}$ and $v=-\frac{\partial \psi}{\partial x}$. Moreover, the momentum and energy equations (2) and (3) are transformed to the ordinary differential equations by defined the following similarity variables (Ishak et al., 2009)

$\psi=\sqrt{v u_{w} x} f(\eta), \eta=\sqrt{\frac{u_{w}}{v x}} y, T=T_{\infty}+\frac{b x}{1-c t} \theta(\eta)$

where $f(\eta)$ is the non-dimensional stream function, $\eta$ is the similarity variable and $\theta(\eta)$ is the non-dimensional temperature. The converted equations are

$f^{\prime \prime \prime}+f f^{\prime \prime}-A\left(\frac{1}{2} \eta f^{\prime \prime}+f^{\prime}\right)-f^{\prime 2}-M f^{\prime}=0$ 
International Journal of Mathematical, Engineering and Management Sciences

Vol. 4, No. 4, 1018-1030, 2019

https://dx.doi.org/10.33889/IJMEMS.2019.4.4-081

$\theta^{\prime \prime}+\operatorname{Pr}\left[f \theta^{\prime}-A\left(\frac{1}{2} \eta \theta^{\prime}+\theta\right)-f^{\prime} \theta\right]=0$

with the associated boundary conditions

$\begin{array}{ll}f=S, f^{\prime}=1, \theta=1 & \text { at } \eta=0 \\ f^{\prime} \rightarrow 0, \theta \rightarrow 0 & \text { as } \eta \rightarrow \infty\end{array}$

where primes (') denote differentiation with respect to $\eta, A=\frac{c}{a}$ is the unsteadiness parameter, $M=\frac{\sigma_{e} \mu_{e}{ }^{2} H_{0}{ }^{2} v \operatorname{Re}}{\rho u_{w}{ }^{2}}$ is the magnetic parameter, $\operatorname{Re}=\frac{u_{w} x}{v}$ is the local Reynolds number and $\operatorname{Pr}=\frac{v}{\alpha}$ is the Prandtl number.

\section{Method of Solution}

Apply a perturbation technique for the computational solution of the equations (6) and (7) along to the corresponding boundary conditions equations (8), by surmising the power series in terms of the small magnetic parameter $M$ as

$$
\begin{aligned}
& f(\eta)=\sum_{i=0}^{\infty} M^{i} f_{i}(\eta) \\
& \theta(\eta)=\sum_{j=0}^{\infty} M^{j} \theta_{j}(\eta)
\end{aligned}
$$

Substituting equations (9) and (10) and its derivatives in equations (6) and (7) and comparing the coefficients of like powers of $M$, the following set of equations are found

$$
\begin{aligned}
& f_{0}^{\prime \prime \prime}+f_{0} f_{0}^{\prime \prime}-A\left(\frac{1}{2} \eta f_{0}^{\prime \prime}+f_{0}^{\prime}\right)-f_{0}^{\prime 2}=0 \\
& \theta_{0}^{\prime \prime}+\operatorname{Pr}\left[f_{0} \theta_{0}^{\prime}-A\left(\frac{1}{2} \eta \theta_{0}^{\prime}+\theta_{0}\right)-f_{0}^{\prime} \theta_{0}\right]=0 \\
& f_{1}^{\prime \prime \prime}+f_{0} f_{1}^{\prime \prime}-A\left(\frac{1}{2} \eta f_{1}^{\prime \prime}+f_{1}^{\prime}\right)-2 f_{0}^{\prime} f_{1}^{\prime}+f_{0}^{\prime \prime} f_{1}=f_{0}^{\prime} \\
& \theta_{1}^{\prime \prime}+\operatorname{Pr}\left[f_{0} \theta_{1}^{\prime}-A\left(\frac{1}{2} \eta \theta_{1}^{\prime}+\theta_{1}\right)-f_{0}^{\prime} \theta_{1}\right]=-\operatorname{Pr}\left(f_{1} \theta_{0}^{\prime}-f_{1}^{\prime} \theta_{0}\right) \\
& f_{2}^{\prime \prime \prime}+f_{0} f_{2}^{\prime \prime}-A\left(\frac{1}{2} \eta f_{2}^{\prime \prime}+f_{2}^{\prime}\right)-2 f_{0}^{\prime} f_{2}^{\prime}+f_{0}^{\prime \prime} f_{2}=-f_{1} f_{1}^{\prime \prime}+f_{1}^{\prime 2}+f_{1}^{\prime} \\
& \theta_{2}^{\prime \prime}+\operatorname{Pr}\left[f_{0} \theta_{2}^{\prime}-A\left(\frac{1}{2} \eta \theta_{2}^{\prime}+\theta_{2}\right)-f_{0}^{\prime} \theta_{2}\right]=-\operatorname{Pr}\left(f_{1} \theta_{1}^{\prime}-f_{1}^{\prime} \theta_{1}+f_{2} \theta_{0}^{\prime}-f_{2}^{\prime} \theta_{0}\right)
\end{aligned}
$$

with the relevant boundary conditions 
International Journal of Mathematical, Engineering and Management Sciences

Vol. 4, No. 4, 1018-1030, 2019

https://dx.doi.org/10.33889/IJMEMS.2019.4.4-081

$$
\begin{aligned}
& f_{0}=S, f_{i}=0, f_{0}^{\prime}=1, f_{i}^{\prime}=0, \theta_{0}=1, \theta_{i}=0 ; i>0 \quad \text { at } \eta=0 \\
& f_{j}^{\prime} \rightarrow 0, \theta_{j} \rightarrow 0 ; j \geq 0 \quad \text { as } \eta \rightarrow \infty
\end{aligned}
$$

The equation (11) was obtained by Ishak et al. (2009) in the absence of magnetic impact and the resting equations are solved numerically via Runge-Kutta fourth order method in association with shooting technique (Na, 1979; Hoffman, 2001) with step size 0.001 . The above process is imitated until finding the converged results up to the aimed level of accuracy $10^{-5}$. Consequently, the numerical values of velocity and temperature fields are sketched in Figures 2 to 4 and 5 to 8 respectively for illustrations of the result.

\section{Local Skin Friction Coefficient and Local Nusselt Number}

In addition to velocity and temperature, the physical quantities of engineering interest are the local skin friction coefficient $C_{f}$ and the local Nusselt number $N u$, which in the dimensional form are expressed as

$$
C_{f}=\frac{\tau_{w}}{\frac{\rho u_{w}^{2}}{2}}
$$

$$
N u=\frac{x q_{w}}{\kappa\left(T_{w}-T_{\infty}\right)}
$$

where $\tau_{w}=\mu\left(\frac{\partial u}{\partial y}\right)_{y=0}$ and $q_{w}=-\kappa\left(\frac{\partial T}{\partial y}\right)_{y=0}$ are the wall shear stress and the wall heat flux respectively, for the present case the equations (18) and (19) are obtained as follows

$$
\begin{aligned}
& f^{\prime \prime}(0)=\frac{\sqrt{\operatorname{Re}}}{2} C_{f} \\
& \theta^{\prime}(0)=-\frac{1}{\sqrt{\operatorname{Re}}} N u
\end{aligned}
$$

Numerical values of $f^{\prime \prime}(0)$ and $\theta^{\prime}(0)$ for distinct values of the specified parameters are given in Tables 1 and 2 respectively.

\section{Results Discussion}

Figures 2 to 4 illustrate the velocity profiles $f^{\prime}(\eta)$ for the changeable value of the suction/injection parameter $S$, the unsteadiness parameter $A$ and the magnetic parameter $M$ respectively, along the remaining physical parameters are constant. From these figures, it may be noticed that the fluid flow declines with the enhancement in the values of $S, A$ and $M$, whereas for the value of $\eta>2.5$ Figure 3 exhibits converse behavior. Moreover, the effect of drag force (called Lorentz force) composed through the transverse magnetic field on electrically conducting fluid, causes deceleration of the fluid motion and increase the fluid temperature (Figure 7). 
International Journal of Mathematical, Engineering and Management Sciences

Vol. 4, No. 4, 1018-1030, 2019

https://dx.doi.org/10.33889/IJMEMS.2019.4.4-081

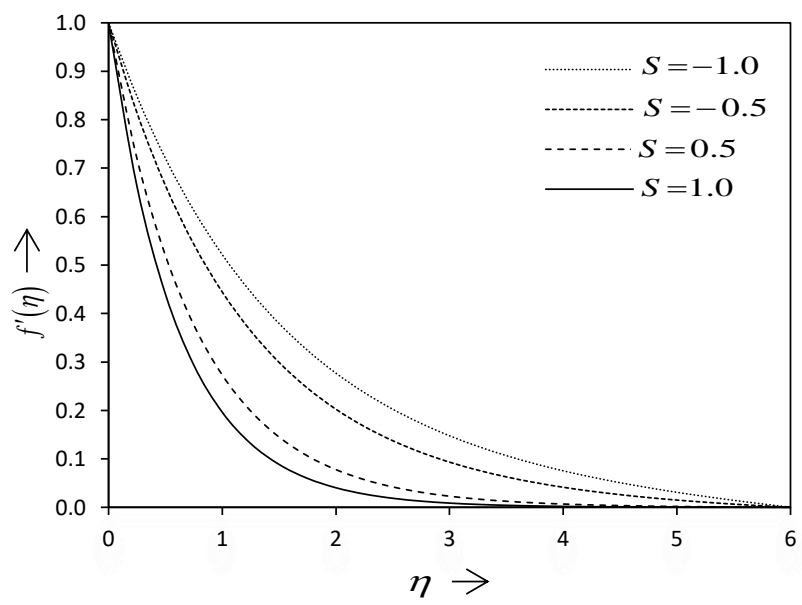

Figure 2. Velocity profiles against $\eta$ for various values of $S$ with $A=0.1$ and $M=0.1$

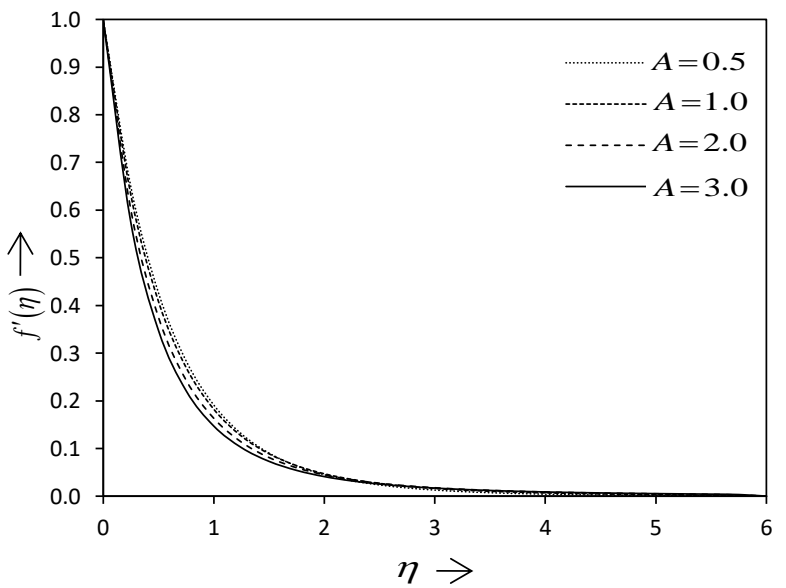

Figure 3. Velocity profiles against $\eta$ for various values of $A$ with $S=1.0$ and $M=0.1$

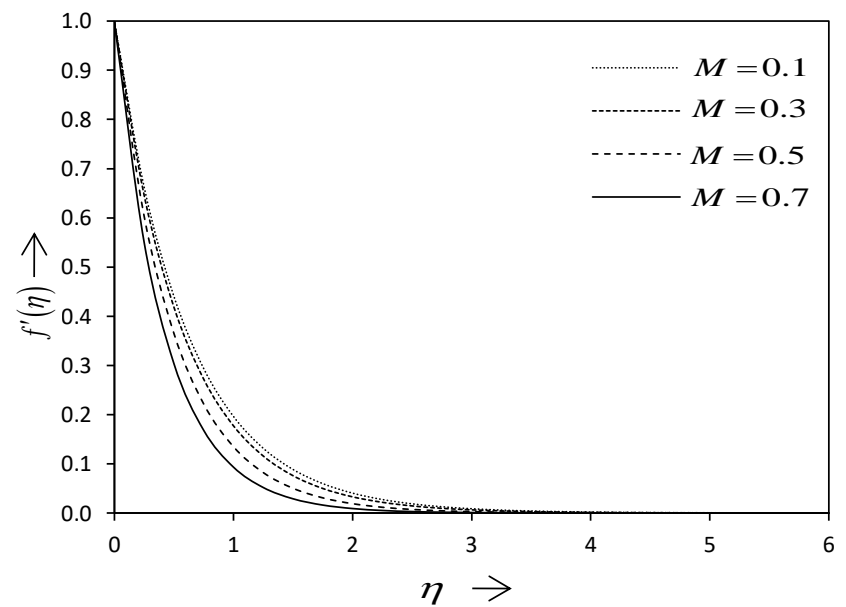

Figure 4. Velocity profiles against $\eta$ for various values of $M$ with $S=1.0$ and $A=0.1$ 
International Journal of Mathematical, Engineering and Management Sciences

Vol. 4, No. 4, 1018-1030, 2019

https://dx.doi.org/10.33889/IJMEMS.2019.4.4-081

The results of the dimensionless temperature $\theta(\eta)$ for various values of $S, A, M$ and $\operatorname{Pr}$ are portrayed in Figures 5 to 8 respectively, while the other physical parameters are fixed. It is evident from these figures that the temperature reduces along with the booming values of $S, A$ and $\operatorname{Pr}$, although the opposite impact is found for $M$ and likewise observed for the value of $\eta>2.5$ as exhibited in Figure 6. Also, Figure 5 represents that the surface heat flux is higher for suction $S>0$ as compared by injection $S<0$. Therefore, suction can be handling for the surface cooling enough faster than injection. Furthermore, the greater Prandtl number fluid has relatively a higher viscosity or a lower thermal conductivity which concludes in the thinner thermal boundary layer, decrease in the fluid temperature and hence, a higher rate of heat transfer at surface. Also, for greater Prandtl number, the thermal boundary layer falls down at a faster rate. This leads that the cooling rate is faster for greater Prandtl number. Therefore, the Prandtl number can be handling to evolve the cooling rate in resulting in fluid flows.

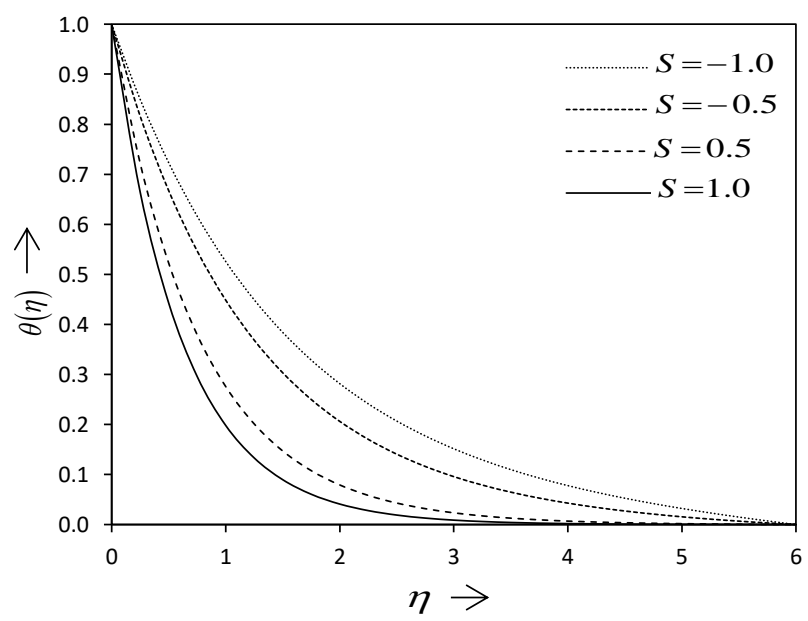

Figure 5. Temperature profiles against $\eta$ for various values of $S$ with $A=0.1, M=0.1$ and $\operatorname{Pr}=1.0$

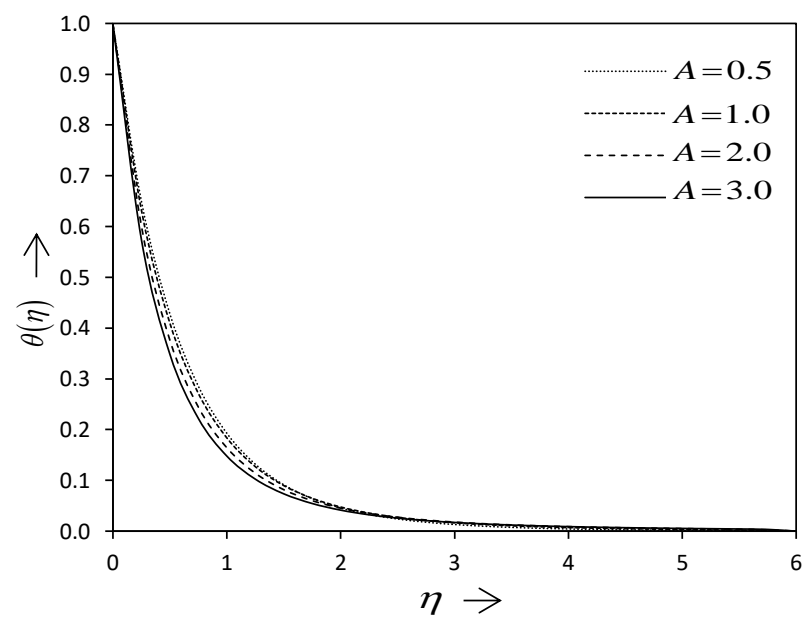

Figure 6. Temperature profiles against $\eta$ for various values of $A$ with $S=1.0, M=0.1$ and $\operatorname{Pr}=1.0$ 
International Journal of Mathematical, Engineering and Management Sciences

Vol. 4, No. 4, 1018-1030, 2019

https://dx.doi.org/10.33889/IJMEMS.2019.4.4-081

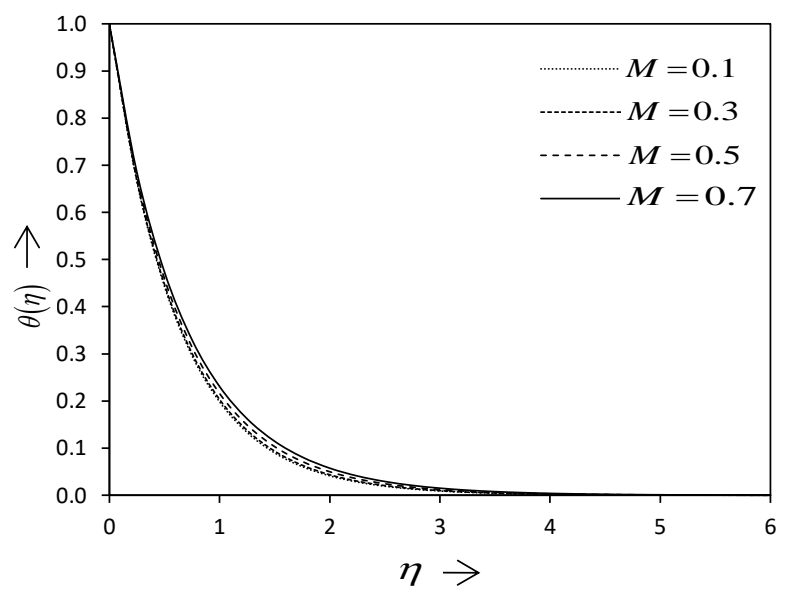

Figure 7. Temperature profiles against $\eta$ for various values of $M$ with $S=1.0, A=0.1$ and $\operatorname{Pr}=1.0$

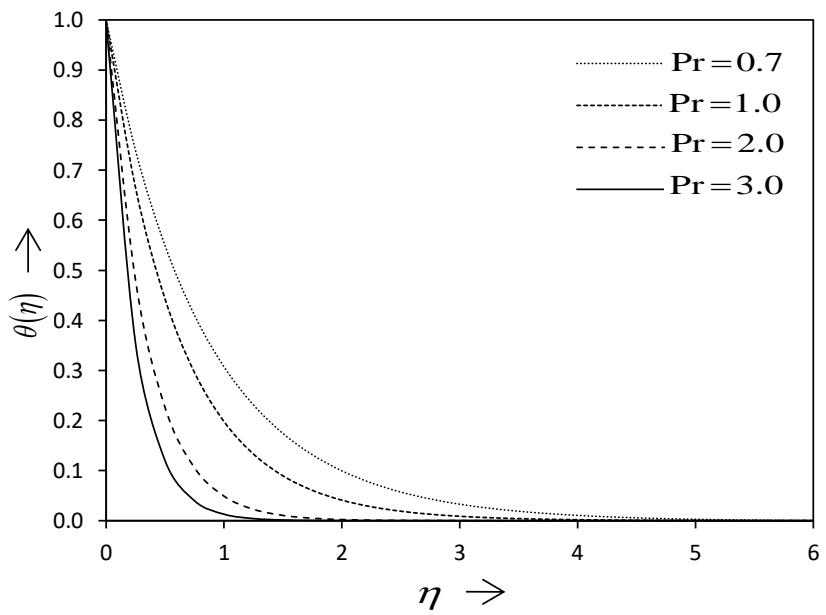

Figure 8. Temperature profiles against $\eta$ for various values of $\operatorname{Pr}$ with $S=1.0, A=0.1$ and $M=0.1$

Table 1 illustrates the local skin friction coefficient $C_{f}$ in terms of the wall shear stress (or the surface gradient) $f^{\prime \prime}(0)$ for several values of $S, A$ and $M$. Table indicated that $C_{f}$ reduces over the raising values of $S, A$, and $M$ taking other physical parameters constant. Besides, it is noted that the values of $f^{\prime \prime}(0)$ are negative for impacts of all physical parameters considered. In the physical fact, negative value of surface gradient leads that the surface wields a drag force on the fluid.

Table 2 depicts the effects of $S, A, M$ and $\operatorname{Pr}$ on the heat transfer rate (or the temperature gradient) $\theta^{\prime}(0)$ at the surface. The comparison of the present results, with the results obtained by Ishak et al. (2009), are found concordant. The table represents that the local Nusselt number $\mathrm{Nu}$ declines with enhancing values of $S, A$ and $\operatorname{Pr}$, whereas, the opposite phenomenon occurs for 
International Journal of Mathematical, Engineering and Management Sciences

Vol. 4, No. 4, 1018-1030, 2019

https://dx.doi.org/10.33889/IJMEMS.2019.4.4-081

$M$, taking other physical parameters fixed. It is also detected that the temperature gradient is negative for all physical parameters specified, which denotes that there is a heat transfer from the surface.

Table 1. Values of $f^{\prime \prime}(0)$ for various values of $S, A$ and $M$

\begin{tabular}{|c|c|c|c|}
\hline$S$ & $A$ & $M$ & $-f^{\prime \prime}(0)$ \\
\hline-1.0 & 0.1 & 0.1 & 0.628175 \\
\hline-0.5 & & & 0.791421 \\
\hline 0.5 & & & 1.285129 \\
\hline 1.0 & & & 1.617678 \\
\hline & 0.5 & & 1.721770 \\
\hline & 1.0 & & 1.849368 \\
\hline & 2.0 & & 2.086832 \\
\hline & 3.0 & 0.3 & 2.299925 \\
\hline & 0.1 & 0.5 & 1.718507 \\
\hline & & 0.7 & 1.990376 \\
\hline
\end{tabular}

Table 2. Comparison of $-\theta^{\prime}(0)$ for various values of $S, A, M$ and $\operatorname{Pr}$

\begin{tabular}{|c|c|c|c|c|c|}
\hline$S$ & $A$ & $M$ & $\operatorname{Pr}$ & Ishak et al. (2009) & Present Results \\
\hline-1.5 & 1.0 & 0.0 & 1.0 & 0.8095 & 0.809571 \\
\hline 0.0 & & & & 1.3205 & 1.320640 \\
\hline 1.5 & & & & 2.2224 & 2.222548 \\
\hline-1.0 & 0.1 & 0.1 & & & 0.621232 \\
\hline-0.5 & & & & & 0.783041 \\
\hline 0.5 & & & & & 1.274117 \\
\hline \multirow[t]{12}{*}{1.0} & & & & & 1.605432 \\
\hline & 0.5 & & & & 1.709830 \\
\hline & 1.0 & & & & 1.837760 \\
\hline & 2.0 & & & & 2.075527 \\
\hline & 3.0 & & & & 2.288617 \\
\hline & 0.1 & 0.3 & & & 1.590010 \\
\hline & & 0.5 & & & 1.550880 \\
\hline & & 0.7 & & & 1.503348 \\
\hline & & 0.1 & 0.7 & & 1.190688 \\
\hline & & & 1.0 & & 1.605432 \\
\hline & & & 2.0 & & 2.857070 \\
\hline & & & 3.0 & & 4.011680 \\
\hline
\end{tabular}

\section{Concluding Remarks}

A model for the numerical study is developed to investigate the analysis of the heat transfer on unsteady boundary layer flow of an incompressible viscous and electrically conducting fluid past a stretchable permeable surface. Employ the similarity transformations to convert the governing partial differential equations to ordinary differential equations. Finally, the numerical solutions of the set of resulting equations are found by implementing the Runge-Kutta fourth order method in association with the shooting technique. The numerical results for the surface heat flux are in congruous with the earlier results in the absence of the magnetic parameter. In present work, it is 
International Journal of Mathematical, Engineering and Management Sciences

Vol. 4, No. 4, 1018-1030, 2019

https://dx.doi.org/10.33889/IJMEMS.2019.4.4-081

observed that the fluid flow, as well as the surface gradient, depreciates along with the raising values of the suction/injection parameter, the unsteadiness parameter and the magnetic parameter. Subsequently, the fluid velocity enlarges for a booming value of the unsteadiness parameter for $\eta>2.5$. Also, the thickness of thermal boundary layer as well as the temperature gradient stepsdown over the developing nature of the suction/injection parameter, the unsteadiness parameter, and the Prandtl number, whereas these increase along to the increasing values of the magnetic parameter. Furthermore, the fluid temperature boosts with an evolvement in the value of the unsteadiness parameter for $\eta>2.5$.

\section{Conflict of Interest}

Authors declare that they have no conflict of interest.

\section{Acknowledgement}

The authors wish to express their sincere appreciation to the learned referee for careful reading of the manuscript and valuable comments.

\section{References}

Andersson, H.I. (2002). Slip flow past a stretching surface. Acta Mechanica, 158(1-2), 121-125.

Beavers, G.S., \& Joseph, D.D. (1967). Boundary conditions at a naturally permeable wall. Journal of Fluid Mechanics, 30(1), 197-207.

Chaudhary, S., \& Choudhary, M.K. (2018). Partial slip and thermal radiation effects on hydromagnetic flow over an exponentially stretching surface with suction or blowing. Thermal Science, 22(2), 797-808.

Chaudhary, S., \& Kumar, P. (2015). Magnetohydrodynamic stagnation point flow past a porous stretching surface with heat generation. Indian Journal of Pure \& Applied Physics, 53(5), 291-297.

Chaudhary, S., Choudhary, M.K., \& Sharma, R. (2015). Effects of thermal radiation on hydromagnetic flow over an unsteady stretching sheet embedded in a porous medium in the presence of heat source or sink. Meccanica, 50(8), 1977-1987.

Chaudhary, S., Kanika, KM, \& Choudhary, M.K. (2018). Newtonian heating and convective boundary condition on MHD stagnation point flow past a stretching sheet with viscous dissipation and Joule heating. Indian Journal of Pure \& Applied Physics, 56(2), 931-940.

Crane, L.J. (1970). Flow past a stretching plate. Zeitschrift für Angewandte Mathematik und Physik ZAMP, 21(4), 645-647.

Dey, D. (2019). Mixed convective MHD micro-polar fluid flow in a porous medium with radiation absorption. International Journal of Mathematical, Engineering and Management Sciences, 4(2), 387399.

Dutta, S., Sharma, B., \& Hazarika, G.C. (2019). Effects of variable viscosity and thermal conductivity on micropolar fluid flow due to a stretching cylinder in presence of magnetic field. International Journal of Mathematical, Engineering and Management Sciences, 4(3), 745-760.

Elbashbeshy, E.M.A., \& Bazid, M.A.A. (2004). Heat transfer over an unsteady stretching surface. Heat and Mass Transfer, 41(1), 1-4. 
International Journal of Mathematical, Engineering and Management Sciences

Vol. 4, No. 4, 1018-1030, 2019

https://dx.doi.org/10.33889/IJMEMS.2019.4.4-081

Elbashbeshy, E.M., \& Aldawody, D.A. (2010). Heat transfer over an unsteady stretching surface with variable heat flux in the presence of a heat source or sink. Computers \& Mathematics with Applications, 60(10), 2806-2811.

Ghadikolaei, S.S., Hosseinzadeh, K., Ganji, D.D., \& Jafari, B. (2018). Nonlinear thermal radiation effect on magneto Casson nanofluid flow with Joule heating effect over an inclined porous stretching sheet. Case Studies in Thermal Engineering, 12, 176-187.

Hayat, T., \& Sajid, M. (2007). Analytic solution for axisymmetric flow and heat transfer of a second grade fluid past a stretching sheet. International Journal of Heat and Mass Transfer, 50(1-2), 75-84.

Hoffman, J.D. (2001). Numerical Methods for Engineers and Scientists. Second Edition, Marcel Dekker, New York.

Ishak, A., Nazar, R., \& Pop, I. (2008). Heat transfer over an unsteady stretching surface with prescribed heat flux. Canadian Journal of Physics, 86(6), 853-855.

Ishak, A., Nazar, R., \& Pop, I. (2009). Heat transfer over an unsteady stretching permeable surface with prescribed wall temperature. Nonlinear Analysis: Real World Applications, 10(5), 2909-2913.

Jat, R.N., \& Chaudhary, S. (2009). Unsteady magnetohydrodynamic boundary layer flow over a stretching surface with viscous dissipation and Joule heating. Nuovo Cimento della Societa Italiana di Fisica. B, General Physics, Relativity, Astronomy and Mathematical Physics and Methods, 124(1), 53-59.

Khader, M.M., \& Megahed, A.M. (2014). Numerical solution for the flow and heat transfer due to a permeable stretching surface embedded in a porous medium with a second-order slip and viscous dissipation. The European Physical Journal Plus, 129(1), 10.

Liao, S.J. (2007). A new branch of solutions of boundary-layer flows over a permeable stretching plate. International Journal of Non-Linear Mechanics, 42(6), 819-830.

Mabood, F., \& Khan, W.A. (2016). Analytical study for unsteady nanofluid MHD Flow impinging on heated stretching sheet. Journal of Molecular Liquids, 219, 216-223.

Magyari, E., \& Keller, B. (2000). Exact solutions for self-similar boundary-layer flows induced by permeable stretching walls. European Journal of Mechanics-B/Fluids, 19(1), 109-122.

Maity, S., Singh, S.K., \& Kumar, A.V. (2016). Unsteady three dimensional flow of Casson liquid film over a porous stretching sheet in the presence of uniform transverse magnetic field and suction/injection. Journal of Magnetism and Magnetic Materials, 419, 292-300.

Mukhopadhyay, S., \& Layek, G.C. (2012). Effects of variable fluid viscosity on flow past a heated stretching sheet embedded in a porous medium in presence of heat source/sink. Meccanica, 47(4), 863-876.

Na, T.Y. (1979). Computational methods in engineering boundary value problems. Academic Press, New York.

Pandya, N., Srivastava, P.K., Yadav, R., \& Pandey, R.P. (2019). Magneto-hydrodynamic flow of a viscous fluid in a channel with a porous bounding wall of different permeabilities. International Journal of Mathematical, Engineering and Management Sciences, 4(3), 708-728.

Ram, P., Joshi, V.K., \& Makinde, O.D. (2017). Unsteady convective flow of hydrocarbon magnetite nanosuspension in the presence of stretching effects. In Defect and Diffusion Forum (Vol. 377, pp. 155-165). Trans Tech Publications.

Ram, P., Joshi, V.K., Sharma, S., \& Yadav, N. (2018). Boundary layer flow of magnetic nanoliquids due to a radially rotating stretchable plate. In Materials Science Forum (Vol. 928, pp. 100-105). Trans Tech Publications. 
International Journal of Mathematical, Engineering and Management Sciences

Vol. 4, No. 4, 1018-1030, 2019

https://dx.doi.org/10.33889/IJMEMS.2019.4.4-081

Rashad, A.M. (2014). Effects of radiation and variable viscosity on unsteady MHD flow of a rotating fluid from stretching surface in porous medium. Journal of the Egyptian Mathematical Society, 22(1), 134142.

Sakiadis, B.C. (1961). Boundary-layer behavior on continuous solid surfaces: I. Boundary-layer equations for two-dimensional and axisymmetric flow. AIChE Journal, 7(1), 26-28.

Tian, X.Y., Li, B.W., \& Zhang, J.K. (2017). The effects of radiation optical properties on the unsteady 2D boundary layer MHD flow and heat transfer over a stretching plate. International Journal of Heat and Mass Transfer, 105, 109-123.

Wang, C.Y. (1990). Liquid film on an unsteady stretching surface. Quarterly of Applied Mathematics, 48(4), 601-610. 